\title{
Survey of Cherry necrotic rusty mottle virus and Cherry green ring mottle virus incidence in Korea by Duplex RT-PCR
}

\author{
Seung-Yeol Lee ${ }^{1}$, Mi-Chi Yea ${ }^{1,3}$, Chang-Gi Back ${ }^{1}$, Kwang-Shik Choi ${ }^{3}$, In-Kyu Kang ${ }^{1}$, Su-Heon Lee ${ }^{1}$ and \\ Hee-Young Jung ${ }^{1 *}$ \\ ${ }^{1}$ College of Agriculture and Life Sciences, Kyungpook National University, Daegu 702-701, Korea \\ ${ }^{2}$ Animal, Plant and Fisheries Quarantine and Inspection Agency, 433-1 Anyang 430-016, Korea \\ ${ }^{3}$ College of Natural Sciences, Kyungpook National University, Daegu 702-701, Korea
}

(Received on May 7, 2014; Revised on July 24, 2014; Accepted on August 12, 2014)

The incidence of Cherry necrotic rusty mottle virus (CNRMV) and Cherry green ring mottle virus (CGRMV) have recently been occurred in Korea, posing a problem for sweet cherry cultivation. Since infected trees have symptomless leaves or ring-like spots on the pericarp, it is difficult to identify a viral infection. In this study, the incidence of CNRMV and CGRMV in sweet cherry in Gyeongbuk province was surveyed using a newly developed duplex reverse transcriptase polymerase chain reaction (RT-PCR) method that can detect both viruses in a single reaction. CNRMV and CGRMV co-infection rates were $29.6 \%, 53.6 \%$, and $17.6 \%$, respectively, in samples collected from three different sites (Daegu, Gyeongju and Gyeongsan) in Gyeongbuk province during 2012 and 2013. This duplex RT-PCR method offers a simple, rapid, and effective way of identifying CNRMV and CGRMV simultaneously in sweet cherry trees, which can aid in the management of viral infections that could undermine yield.

Keywords : CNRMV, CGRMV, Duplex RT-PCR, Infection rate

Cherry necrotic rusty mottle virus (CNRMV) and the closely related Cherry green ring mottle virus (CGRMV) are flexuous filamentous plant viruses and members of Betaflexiviridae (Adams et al., 2004). CNRMV infects stone fruit, including peach, apricot, sour cherry, and sweet cherry, and has been reported in North America, New Zealand, Europe, Chile, Japan, China, and Korea (Fiore and Zamorano, 2013; Isogai et al., 2004; Lee et al., 2012;

*Corresponding author.

Phone) +82-53-950-5760, FAX) +82-53-950-6758

E-mail) heeyoung@knu.ac.kr
Mandic et al., 2007; Wadley and Nyland, 1976; Zhou et al., 2013). CNRMV-infected plants typically develop brown, angular, necrotic spots, rusty chlorotic, and shot hole symptoms on leaves as well as blisters, gum pockets, and general necrosis on the bark (Rott and Jelkmann, 2001). CGRMV also infects stone fruits including sour cherry and oriental flowering cherry, with sweet cherry, peach, and apricot being symptomless hosts, and has a geographic distribution similar to CNRMV (Fiore and Zamorano, 2013; Isogai et al., 2004; Lee et al., 2012; Mandic et al., 2007; Parker et al., 1976; Zhou et al., 2011). CGRMVinfected Montmorency sour cherry shows yellow mottling and ring-like bands on its leaves, and produces misshapen, bitter, and unmarketable fruit (Parker et al., 1976).

In 2011, CNRMV and CGRMV were detected in sweet cherries in Korea (Lee et al., 2012), and abnormal cherries exhibiting ring spots on the pericarp collected from the cities of Daegu, Gyeongju and Gyeongsan were diagnosed as being co-infected with CNRMV and CGRMV (Lee et al., 2014). Asymptomatic cherry leaves from Gyeonggi province and Yeongju city harbored single infections of CNRMV and CGRMV, respectively (Cho et al., 2013; Cho et al., 2014). These reports highlight the difficulty of identifying typical symptoms of CNRMV/CGRMV by naked eye to diagnose an infection at early stages (Cho et al., 2013; Lee et al., 2012).

CNRMV and CGRMV have a similar morphology and genomic organization and share approximately $60 \%$ homology at the nucleotide level (Li and Mock, 2005). Although reverse transcriptase polymerase chain reaction (RT-PCR) methods have been developed to detect CNRMV and CGRMV using specific primer pairs (Li and Mock, 2005; Park and Kim, 2004; Rott and Jelkmann, 2001), an accurate method for detecting both viruses simultaneously would be useful for making rapid and reliable diagnoses. In the present study, a duplex RT- 
PCR method was developed that allows the simultaneous detection of CNRMV and CGRMV in sweet cherries, which was used to survey the incidence of these viruses in Gyeongbuk province, Korea.

Cherry fruits with visible ring-spots or ring spot-like symptoms on the pericarp were randomly collected from sweet cherry (cv. Satonishiki) orchards located in the cities of Daegu, Gyeongju, and Gyeongsan during 2012-2013. Total RNA was extracted from samples using the RNeasy Plant Mini Kit (Qiagen, Valencia, CA, USA) according to the manufacturer's instructions and used for RT-PCR, with CNRMV and/or CGRMV infection confirmed using the primer pair prCN4f (5'-AGC ACW RTT AGG AGC TAC TG-3') and prCN4r (5'-TAT CAT TCA TCA CCA CCA AT- $3^{\prime}$ ) that can amplify both viruses (Park and Kim, 2004). The amplification was performed using the OneStep RT-PCR kit (Solgent, Daejeon, Korea) using previously reported reaction conditions (Park and Kim, 2004) on a Veriti 96-well Thermal Cycler (Applied Biosystems, Carlsbad, CA, USA). DNA fragments of the expected size (400 bp) were amplified from 43 out of 83 samples (data not shown). The two pairs of primers for the duplex reaction were designed for specificity and compatibility based on whole coat protein sequences for CNRMV and CGRMV retrieved from GenBank of the National Center for Biotechnology Information (NCBI). Specific primers for CNRMV (CNSp-F, 5'-CTT TGA TCC CAA AAA TCC CA-3' and CNSp-R, 5'-TGG TYT TGT CAC TTG AAC TGT T-3') and CGRMV (CGSp-F, 5'-GCG CAA ACG GAC CCT AAG-3' and CGSp-R, 5'-CGC CAG TCA CTT CAG TCA TT-3') were designed to amplify fragments of $\sim 210$ and $650 \mathrm{bp}$, respectively, and each were used in simplex RT-PCR reactions to confirm the specificity of the products amplified by duplex RT-PCR. Three representative total RNA samples which confirmed as positive for CNRMV and CGRMV (Lee et al., 2014) were used in both simplex and duplex RT-PCR. Also those samples were retrieved from NCBI (Accession no. CNRMV; AB871372-AB871381 (10 strains), CGRMV; AB863720-AB863734 (15 strains)). For simplex and duplex RT-PCR, the $20-\mu 1$ reaction contained $0.5 \mu$ l total RNA, $4 \mu 15 \times$ RT buffer (Solgent), $1 \mu 1$ of 10 mM dNTP, 1 $\mu \mathrm{l}$ forward and reverse primers (10 pM), $2 \mu \mathrm{l}$ RT (Solgent), and $11.5 \mu \mathrm{R}$ Rase-free water with amplification conditions as follows: reverse transcription at $50^{\circ} \mathrm{C}$ for $30 \mathrm{~min}$, followed by $95^{\circ} \mathrm{C}$ for $15 \mathrm{~min} ; 35$ cycles of $95^{\circ} \mathrm{C}$ for $30 \mathrm{~s}$, $60^{\circ} \mathrm{C}$ for $45 \mathrm{~s}$, and $72^{\circ} \mathrm{C}$ for $45 \mathrm{~s}$; and $72^{\circ} \mathrm{C}$ for $5 \mathrm{~min}$. RTPCR products were visualized on a $1 \%$ agarose gel under UV light after staining with ethidium bromide. Simplex RT-PCR products of expected sizes were observed for



Fig. 1. Simplex RT-PCR for the detection of CNRMV or CGRMV in sweet cherry isolates from Daegu, Gyeongju, and Gyeongsan. Lanes 1, 2, 3, and 7: using a CNRMV-specific primer pair (210 bp), Lanes 4, 5, 6, and 8: using a CGRMVspecific primer pair (650 bp). Lane 1 and 4: Daegu isolates, Lane 2 and 5: Gyeongju isolates, Lane 3 and 6: Gyeongsan isolates, Lane 7 and 8: Healthy cherry leaves (control). M: 100 bp DNA ladder. Arrowheads designated $\mathrm{CG}$ and $\mathrm{CN}$ represent partially amplified coat protein genes from CGRMV and CNRMV, respectively.

positive samples but not for healthy leaf samples (Fig. 1). Amplicons were then purified using the HiGene Gel \& PCR Purification System (Biofact, Daejeon, Korea) and sequenced yielding 176 and $623 \mathrm{bp}$ sequences from each of amplified DNA fragments. A search in GenBank (NCBI) showed that the obtained $176 \mathrm{bp}$ sequences for CNRMV had 99-100\% identity to Daegu (AB871372), Gyeongju (AB871375), and Gyeongsan (AB871380) strains and 685 bp sequences for CGRMV had 99-100\% identity to Daegu (AB863720), Gyeongju (AB863731), and Gyeongsan (AB863734) strains in nucleotide level, respectively. After duplex RT-PCR, DNA fragments of 200 and $650 \mathrm{bp}$ (corresponding to CNRMV and CGRMV, respectively) were detected in each sample by gel electrophoresis (Fig. 2). Also, 10-fold serial dilutions $\left(10^{0}-10^{-4}\right)$ were prepared

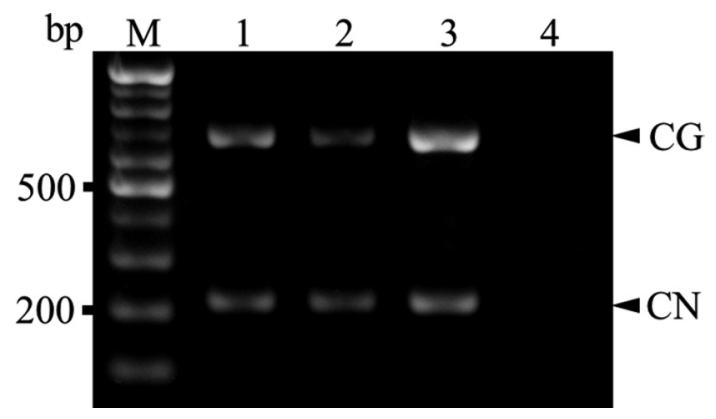

Fig. 2. Duplex RT-PCR for the simultaneous detection of CNRMV and CGRMV in sweet cherry isolates from three Korean cities. Lane 1: Daegu isolate, Lane 2: Gyeongsan isolate, Lane 3: Gyeongju isolate, Lane 4: Healthy cherry leaf (control). M: 100 bp DNA ladder. Arrowheads designated CG and CN represent partially amplified coat protein genes from CGRMV and CNRMV, respectively. 


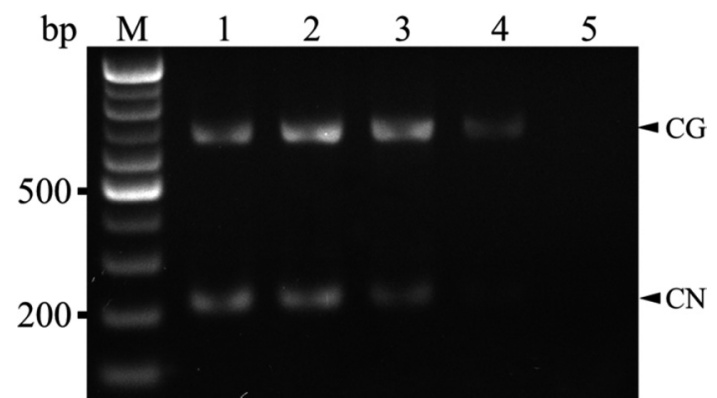

Fig. 3. Duplex RT-PCR detection limits for CNRMV and CGRMV. Total RNA was prepared as 10-fold serial dilutions. Lanes 1-5: $10^{0}-10^{-4}$. M: 100 bp DNA ladder. Arrowheads designated $\mathrm{CG}$ and $\mathrm{CN}$ represent partially amplified coat protein genes from CGRMV and CNRMV, respectively.

from $\sim 10 \mathrm{ng}$ total RNA to determine the detection limits of the duplex RT-PCR. According to our results it was confirmed that it can detect up to $10^{-2}$ and $10^{-3}$ dilution for CNRMV and CGRMV, respectively (Fig. 3).

A total of 43 total RNA samples (9 from Daegu, 20 from Gyeongju, and 14 from Gyeongsan) from abnormal cherries were examined using the developed duplex RTPCR method (Fig. 4A-C). Of these, 11 were positive for CNRMV infection, 4 were infected with CGRMV, and 28 samples were co-infected with both viruses (Table 1). Among Daegu isolates, 8 were co-infected with CNRMV and CGRMV while only 1 sample had a single infection of
CGMRV (Table 1). Of the 20 samples from Gyeongju, 15 were confirmed as being infected by both viruses, making this the city with the highest co-infection rate. Among Gyeongsan isolates, 7 were CNRMV and 2 were CGRMV infections, and 5 were confirmed as co-infections. Thus, the duplex RT-PCR found co-infection rates in Daegu, Gyeongju, and Gyeongsan of $29.6 \%, 53.6 \%$, and $17.9 \%$, respectively. These results demonstrate that CNRMV and CGRMV are more prevalent in these cities than in other surveyed regions in Korea (Table 1; Cho et al., 2013, 2014). In addition, cases of single infection of sweet cherry by CNRMV or CGRMV have been reported in Gyeonggi province and the Yeongju region of Korea; however, coinfections by both viruses have not been observed (Cho et al., 2013, 2014).

To date, several RT-PCR-based methods have been developed to detect CNRMV and/or CGRMV infections in cherry (Rott and Jelkmann, 2001; Isogai et al., 2004; Park et al., 2004; Fiore and Zamorano, 2013). These methods are suitable to detect CNRMV or CGRMV; however, it is impossible to differentiate between the two targeted viruses in single reaction. Multiplex RT-PCR methods are rapid, reliable, and sensitive, and have been widely used to identify more than one virus simultaneously in a single assay, for instance in viral infections of stone fruit (Sanchez-Navarro et al., 2005; Jarosová and Kundu 2010; Yardimci and Çulal-K1llç, 2011) and lily (Kwon et al.,

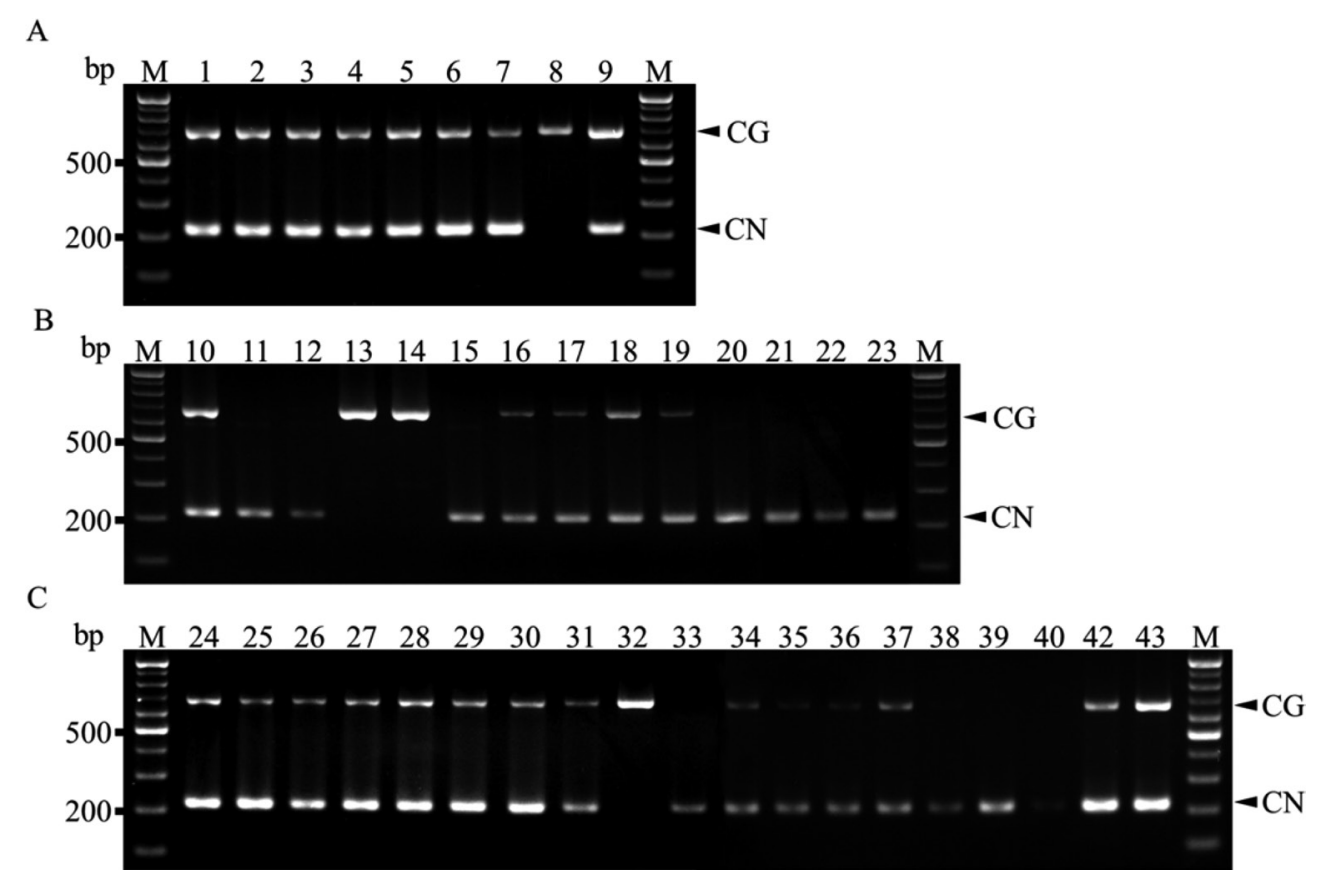

Fig. 4. Simultaneous detection of CNRMV and CGRMV in sweet cherry collected from Daegu, Gyeongsan, and Gyeongju by duplex RT-PCR. A: Daegu isolates (lanes 1-9); B: Gyeongsan isolates (lanes 10-23); C: Gyeongju isolates (lanes 24-43). M: 100 bp DNA ladder. Arrowheads designated CG and CN represent partially amplified coat protein genes from CGRMV and CNRMV, respectively. 
Table 1. Incidence of CNRMV and CGRMV infection in sweet cherry cv. Satonishiki samples 1 collected from Daegu, Gyeongju, and Gyeongsan city in Korea

\begin{tabular}{|c|c|c|c|c|c|c|c|}
\hline \multirow{2}{*}{ Location } & \multirow{2}{*}{ No. of samples } & \multicolumn{3}{|c|}{ No. of infected samples } & \multicolumn{3}{|c|}{ Percent (\%) } \\
\hline & & CNRMV & CGRMV & Both & CNRMV & CGRMV & Both \\
\hline Daegu & 27 & 0 & 1 & 8 & 0 & 3.7 & 29.6 \\
\hline Gyeongju & 28 & 4 & 1 & 15 & 14.3 & 3.6 & 53.6 \\
\hline Gyeongsan & 28 & 7 & 2 & 5 & 25 & 7.1 & 17.9 \\
\hline Total & 83 & 11 & 4 & 28 & 13.3 & 4.8 & 33.7 \\
\hline
\end{tabular}

2013). Multiplex RT-PCR has also been applied to the detection of CNRMV and/or CGRMV (Noorani et al., 2013; Zhao et al., 2013), including in sweet cherries. For CNRMV, specific primers were designed based on the replicase gene and have identified stone fruit viruses such as Cherry virus A, Little cherry virus-1, and Prune necrotic ring spot virus, in addition to CNRMV (Noorani et al., 2013). A duplex RT-qPCR method was also developed for Apple chlorotic leaf spot virus and CGRMV in peach (Zhao et al., 2013).

Co-infections by CNRMV and CGRMV have previously been confirmed in cherry orchards located in Daegu, Gyeongju, and Gyeongsan regions (Lee et al., 2014). Furthermore, it was confirmed that single infections were also occurred in Daegu, Gyeongju, and Gyeongsan city. Cho et al (2013 and 2014) also reported that single infection rate of CNRMV and CGRMV were 1.9\% (3 infected samples out of 154 samples) and 3.9\% (6 infected samples out of 154 samples) respectively, and there was no evidence of mixed infection. In this study, we confirmed $13.3 \%$ and $4.8 \%$ single infection rate while $33.7 \%$ mixed infection rate of CNRMV and CGRMV (Table 1). The viral infection rate especially mixed infection of CNRMV and CGRMV is more prevalent in different cities of Gyeongbuk province (Daegu, Gyeongsan, and Gyeongju city) as compared to infection rate in Gyeonggi province reported by (Cho et al., 2013; 2014). Sweet cherries were firstly introduced in Daegu and Gyeongju city where the cultivation of sweet cherry had begun in Korea. At this time, viral infected cherries could be introduced in Korea. From those orchards, many infected cherry seedlings could be spread around Daegu and Gyeongju city. Hence it is assumed that co-infection with CNRMV and CGRMV was prevalent in Daegu, Gyeongju city compare to Gyeongsan city or Gyeonggi province. Meanwhile, we also checked CNRMV and CGRMV infection rate in two cherry varieties (cvs. Skeena and Regina) collected from orchards located in Daegu city by using CNRMV/CGRMV-specific primer pair (Park et al., 2004) as well as the duplex RT-PCR method. However, there was no evidence of infection by CNRMV and CGRMV in these varieties (data not shown). Meanwhile, it was reported that not only CNRMV and CGRMV but also Cherry virus $A$ (CVA) and Apple chlorotic leaf spot virus (ACLSV) also can infect sweet cherry in Korea (Cho et al., 2013; 2014). Therefore it is necessary to develop new detection methods which can detect and investigate the previous reported viruses in different areas of Korea such as Kimchoen, Hwaseong, Pyeongtaek and so on.

In this study, a method was developed to simultaneously detect CNRMV and CGRMV in a sample by duplex RTPCR, which has not been previously reported in Korea. The results presented here provide useful data on infection rates among sweet cherry cv. Satonishiki in specific regions in Korea, and could be applied to a quarantine system to improve management and selection of virus-free trees.

\section{References}

Adams, M. J., Antoniw, J. F., Bar-Joseph, M., Brunt, A. A., Candresse, T., Foster, G. D., Martelli, G. P., Milne, R. G. and Fauquet, C. M. 2004. The new plant virus family Flexiviridae and assessment of molecular criteria for species demarcation. Arch. Virol. 149:1045-1060.

Cho, I. S., Choi, G. S. and Choi, S. K. 2013. Identification of Cherry green ring mottle virus on sweet cherry trees in Korea. Res. Plant Dis. 19:326-330.

Cho, I. S., Choi, G. S., Choi, S. K., Seo, E. Y. and Lim, H. S. 2014. First report of Cherry necrotic rusty mottle virus infecting sweet cherry trees in Korea. Plant Dis. 98:164.

Fiore, N. and Zamorano, A. 2013. First report of Cherry green ring mottle virus and Cherry necrotic rusty mottle virus in sweet cherry (Prunus avium) in Chile and South America. Plant Dis. 97:1122.

Isogai, M., Aoyagi, J., Nakagawa, M., Kubodera, Y., Satoh, K., Katoh, T., Inamori, M., Yamashita, K. and Yoshikawa, N. 2004. Molecular detection of five cherry viruses from sweet cherry trees in Japan. J. Gen. Plant Pathol. 70:288-291.

Jarosová, J. and Kundu, J. K. 2010. Simultaneous detection of stone fruit tree viruses by one-step multiplex RT-PCR. Sci. 
Hortic. (Amsterdam) 125:68-72.

Kwon, J. Y., Ryu, K. H. and Choi, S. H. 2013. Reverse transcription polymerase chain reaction-based system for simultaneous detection of multiple lily-infection viruses. Plant Pathol. J. 29:338-343.

Lee, S. Y., Back, C. G., Kim, Y. H., Yea, M. C., Lee, S. H., Choi, C. and Jung, H. Y. 2012. First report of occurrence of quarantine viral disease on sweet cherry in Korea. Res. Plant Dis. 18:464 (Abstract).

Lee, S. Y., Yea, M. C., Back, C. G., Choi, C., Lee, S. H., Kang, I. K., Jung, H. Y. and Ohga, S. 2014. Occurrence of Cherry necrotic rusty mottle virus (CNRMV) and Cherry green ring mottle virus on sweet cherry. J. Fac. Agr. Kyushu Univ. 59:14.

Li, R. and Mock, R. 2005. An improved reverse transcriptionpolymerase chain reaction (RT-PCR) assay for the detection of two cherry flexiviruses in Prunus spp. J. Virol. Methods 129:162-169.

Mandic, B., Matić, S., Rwahnih, M. A., Jelkmann, W. and Myrta, A. 2007. Viruses of sweet and sour cherry in Serbia. J. Plant Pathol. 89:103-108.

Noorani, M. S., Awasthi, P., Sharma, M. P., Ram, R. and Zaidi, A. A. 2013. Simultaneous detection and identification of four cherry viruses by two step multiplex RT-PCR with an internal control of plant nad5 mRNA. J. Virol. Methods 193:103-107.

Park, M. R. and Kim, K. H. 2004. RT-PCR detection of three non-reported fruit tree viruses useful for quarantine purpose in Korea. Plant Pathol. J. 20:147-154.
Parker, K. G., Fridland, P. R. and Gilmer, G. M. 1976. Green ring mottle. In: Virus diseases and noninfectious diseases of stone fruit in North America. US Department of Agriculture Handbook No. 437, pp. 193-199.

Rott, M. E. and Jelkmann, W. 2001. Complete nucleotide sequence of Cherry necrotic rusty mottle virus. Arch. Virol., 146:395-401.

Sanchez-Navarro, J. A., Aparicio, F., Herranz, M., Minafra, C., Myrta, A. and Pallas, V. 2005. Simultaneous detection and identification of eight stone fruit viruses by one-step RT-PCR. Eur. J. Plant Pathol. 111:77-84.

Wadley, B. and Nyland, G. 1976. Rusty mottle group. In: Virus diseases and noninfectious diseases of stone fruit in North America. US Department of Agriculture Handbook No. 437, pp. 242-249.

Yardimci, B. C. N. and Çulal-Klllç, H. 2011. Detection of viruses infecting stone fruits in Western Mediterranean region of Turkey. Plant Pathol. J. 27:44-52.

Zhou, J. F., Wang, G. P., Kuang, R. F., Wang, L. P. and Hong, N. 2011. First report of Cherry green ring mottle virus on cherry and peach grown in China. Plant Dis. 95:1319.

Zhou, J. F., Wang, G. P., Qu, L. N., Deng, C. L. Wang, Y., Wang, L. P. and Hong, N. 2013. First report of Cherry necrotic rusty mottle virus on stone fruit trees in China. Plant Dis. 97:290.

Zhao, Z., Yu, Y., Zhang, Z., Liang, P., Ma, Y., Li, S. and Wang, H. 2013. A duplex, SYBR green I-based RT-qPCR assay for the simultaneous detection of Apple chlorotic leaf spot virus and Cherry green ring mottle virus in peach. Virol. J. 10:255. 